\title{
Publisher's Note: Colloidal interactions in a homeotropic nematic cell with different elastic constants [Phys. Rev. E 92, 042505 (2015)]
}

O. M. Tovkach, S. B. Chernyshuk, and B. I. Lev

(Received 9 November 2015; published 23 November 2015)

DOI: 10.1103/PhysRevE.92.059903

PACS number(s): 61.30.Dk, 82.70.Dd, 64.70.M-, 99.10.Fg

This paper was published online on 26 October 2015 with an error in Eq. (19) and an error in the text on page 3. Equation (19) should read as

$$
\begin{aligned}
U_{\mathrm{qq}}^{0}= & -2 \pi K q q^{\prime}\left[\frac{1}{\sqrt{\kappa_{1}}} \frac{1}{\sqrt{\kappa_{3} \rho^{2}+\kappa_{1} l^{2}}}-\frac{\cos 2 \varphi}{\sqrt{\kappa_{1}}} \frac{\left(\sqrt{\kappa_{3} \rho^{2}+\kappa_{1} l^{2}}-\sqrt{\kappa_{1} l^{2}}\right)^{2}}{\kappa_{3} \rho^{2} \sqrt{\kappa_{3} \rho^{2}+\kappa_{1} l^{2}}}\right. \\
& \left.+\frac{1}{\sqrt{\kappa_{2}}} \frac{1}{\sqrt{\kappa_{3} \rho^{2}+\kappa_{2} l^{2}}}+\frac{\cos 2 \varphi}{\sqrt{\kappa_{2}}} \frac{\left(\sqrt{\kappa_{3} \rho^{2}+\kappa_{2} l^{2}}-\sqrt{\kappa_{2} l^{2}}\right)^{2}}{\kappa_{3} \rho^{2} \sqrt{\kappa_{3} \rho^{2}+\kappa_{2} l^{2}}}\right] .
\end{aligned}
$$

The sentence following Eq. (19) should read as "Within the one-constant approximation $\left(\kappa_{s}=1\right)$ Eq. (19) yields a Coulomb-like....." On page 3, the first line of the second paragraph in the right-hand column should read "Now $F_{\text {tot }}$ is the sum of $F_{\text {bulk }}$ and $F_{\text {source }}$ given by (12) and (13) respectively." The equation and text have been corrected as of 9 November 2015. The equation and text are incorrect in the printed version of the journal. 